Proceedings

\title{
A Hybrid Structural Health Monitoring Approach Based on Reduced-Order Modelling and Deep Learning ${ }^{\dagger}$
}

\author{
Luca Rosafalco ${ }^{1, *(\mathbb{D}}$, Alberto Corigliano ${ }^{1}\left(\mathbb{D}\right.$, Andrea Manzoni ${ }^{2}(\mathbb{D})$ and Stefano Mariani ${ }^{1}(\mathbb{D}$ \\ 1 Dipartimento di Ingegneria Civile e Ambientale, Politecnico di Milano, Piazza L. da Vinci 32, 20133 Milano, \\ Italy; alberto.corigliano@polimi.it (A.C.); stefano.mariani@polimi.it (S.M.) \\ 2 MOX, Dipartimento di Matematica, Politecnico di Milano, Piazza L. da Vinci 32, 20133 Milano, Italy; \\ andrea1.manzoni@polimi.it \\ * Correspondence: luca.rosafalco@polimi.it; Tel.: +39-02-23994273 \\ + Presented at the 6th International Electronic Conference on Sensors and Applications, 15-30 November 2019; \\ Available online: https:/ /ecsa-6.sciforum.net/.
}

Published: 14 November 2019

\begin{abstract}
Recent advances in sensor technologies coupled with the development of machine/deep learning strategies are opening new frontiers in Structural Health Monitoring (SHM). Dealing with structural vibrations recorded with pervasive sensor networks, SHM aims at extracting meaningful damage-sensitive features from the data, shaped as multivariate time series, and taking real-time decisions concerning the safety level. Within this context, we discuss an approach able to detect and localize a structural damage avoiding any pre-processing of the acquired data. The method takes advantage of the capability of Deep Learning of Fully Convolutional Networks, trained during an offline SHM phase. As a hybrid model- and data-based solution is looked for, Reduced Order Models are also built in the offline phase to reduce the computational burden of the whole monitoring approach. Through a numerical benchmark test, we show how the proposed method can recognize and localize different damage states.
\end{abstract}

Keywords: structural health monitoring; fully convolutional networks; damage localization; time series analysis; deep learning

\section{Introduction}

Collapses of civil infrastructures can be due to structural deterioration or to working conditions not in compliance with the design ones. SHM is aimed at assessing the safety level of aging structures by localizing and quantifying a damage state, possibly in real-time [1-4]. Since visual inspections are usually not able to provide quantitative estimates of such damage, recent advances in sensing technologies and signal processing are exploited to automatically and robustly monitor the health of real-life structural systems [5].

By attacking the damage detection and localization problem as a classification one [6], to distinguish the current state from the healthy baseline a Simulation-Based Classification approach is adopted [7], ruled by Deep Learning (DL) techniques. Copying with model uncertainties, measurement noise and operational/environmental variability, DL potentially allows classification in an automatic and efficient fashion. As the structural output consists of displacement and/or acceleration recordings, classification aims at recognizing which structural state, belonging to a discrete set, could have most probably produced them. Each damaged state in the mentioned set is characterized by its own damage 
scenario, different from all the others: the larger the number of the considered scenarios, the higher the capability of the method to identify the actual damage location.

Focusing on data acquisition and feature extraction of the SHM strategy, a vibration-based approach is adopted whose strength is due to the fact that, once damage is triggered, the dynamic response of the structure gets modified along with the relevant modal information [8]. By collecting the displacement and/or acceleration histories at ad-hoc designed locations, the approach enables the analysis of global and local structural behaviors, even without any preliminary modal analysis $[9,10]$. Feature extraction can consist in determining the system vibration frequencies and modal shapes, but more involved structural properties or outcomes are to be accounted for to get rid of the spurious effects of operational/environmental variability [11,12].

In the literature, model-based and data-based approaches to (early) damage detection are typically adopted disjointly; in this work we try instead to cope with the SHM problem by exploiting the different features and strengths of the two approaches. By introducing an offline-online decomposition of the procedure, the initial offline stage is performed to train or update the model and to establish a damage-free baseline; the subsequent online stage aims at novelty detection, wherein the novelty is represented by damage occurrence. Data assimilation techniques such as Kalman or particle filters can be employed to efficiently deal with model updating [13]. To reduce the computational burden of dataset construction, simplified or reduced-order models (ROMs) take the place of high-fidelity simulations, characterized by being bias-free but often too accurate for the sole scope of damage detection. Specifically, in this work, the Reduced Basis (RB) method [14,15] is adopted for the construction of reliable and efficient low-fidelity ROMs.

When all the possible damage scenarios have been modeled, Machine Learning tools can perform the classification task in a supervised, unsupervised or semi-supervised way [2]. The adopted supervised DL approach allows data dimensionality reduction and hierarchical pattern recognition $[16,17]$ and is therefore becoming popular for SHM purposes [18,19].

To go through the main features of the proposed SHM strategy, the remainder of this paper is organized as follows. In Section 2 the methodological approach to the SHM problem is detailed, along with the Neural Network (NN) architecture. In Section 3 we briefly summarize the rationale of the adopted RB approach to model order reduction. In Section 4 we describe the numerical benchmark and present the obtained results. Finally, Section 5 gathers some concluding remarks and foresees future developments.

\section{SHM Methodology and Fully Convolutional Networks}

As mentioned, the SHM procedure is subdivided into an offline phase and a subsequent online phase. In the offline phase dataset construction and training of the damage classifier are carried out. The dataset is made of multivariate time series, represented by the output of the sensor network in the different scenarios obtained by selectively reducing the structural stiffness with respect to the reference state. As discussed in [4,5], damage is considered frozen for each solution within the observation interval, so that the structure behaves linearly.

To feed the procedure with effective information on the damage state, the sensor network has to be designed according to e.g., a Bayesian optimization technique [20,21]; this guarantees that the collected measurements, even if affected by instrumental and environmental noise, bring relevant information for damage estimation. The simulated operational conditions and damage scenarios should be thus included in the dataset, according to a statistical criterion [2].

The dataset is used to train a Fully Convolutional Network (FCN) classifier. Classifiers with a different complexity can be employed, and the generalization capacities of the trained FCN must be finally assessed on the test set; additional details can be found in [22,23]. During the online 
phase, the signals acquired by the sensors are provided to the classifier, and damage localization and quantification is performed, handling only a small amount of information to allow real time monitoring.

Since interconnected sensors in a network provide multivariate time series, FCNs can be effectively exploited to extract damage-sensitive features from the time series and analyze them [24]. The adopted FCN has been obtained by stacking three convolutional layers (CLs), each one featuring a different filter size, followed by a global pooling and a softmax classifier. Further to that, each CL has been used together with a Batch-Normalization (BN) layer and a Rectified Linear Unit (ReLU) activation layer [24,25]. In case of time series of different length, for each series the convolutional architecture is adopted separately, and then, data fusion on the extracted features is performed via a concatenation layer. Tensorflow [26] has been used for the NN construction.

While the input channels of the first CL are the displacement and/or acceleration time series recorded by the monitoring system, the outputs, even if still shaped as time series, do not represent the history of the actual structural response any more: They are the so-called features extracted from the input channels. In concrete terms, the tasks performed by the CL are the subdivision of the inputs into data sequences, whose length determines the receptive field and the multiplication of each data sequence by a filter; the output of each filter is called feature map. As the goal of stacking several CLs is to lead to non linear transformations of the input channels, the overall effect is to make the classes linearly separable [27].

A BN layer is introduced after each CL to address the possible issues related to the vanishing/ exploding gradients experienced with DL [28]; for the same reason, the ReLU activation function is preferred to saturating ones [29]. The softmax activation layer finally performs the classification task, estimating the probability that one input channel belongs to a class, or damage scenario.

The NN training consists in the minimization of a loss function. For classification purposes, the commonly adopted loss function is the cross entropy. According to a standard procedure, part of the instances in the dataset (training set) are exploited to train the NN by back-propagating the classification error; a validation set is next employed to test the NN generalization capability and, possibly, to interrupt the training in case of overfitting. A test set is finally employed to verify the prediction capabilities of the trained NN.

The NN hyperparameters, on top of all the dimensions of the kernels and the number of feature maps, have been set according to the following. The number of filters to be adopted depends on the complexity of the classification task. Increasing the number of filters beyond a certain problem-dependent threshold has no effect on the prediction capabilities of the NN, but risks like overfitting of the training dataset can arise. Hence, we have kept $N_{1}=N, N_{2}=2 N$, and $N_{3}=N$ as filter sequence and verified that by increasing $N$ beyond $N=16$ the $N N$ performance does not get affected.

\section{Elastodynamics and Model Order Reduction}

The dataset construction requires the evaluation of the building response for multiple inputs. From a mathematical point of view, such multiple evaluation is equivalent to reconstructing the high-fidelity solution set. To reduce the computational burden, the RB method has been exploited to provide a fast and reliable evaluation of the input-output relationship, thus approximating the high-fidelity solution through a set of RB functions [14]. To construct the RB solution space, a set of high fidelity solutions, called snapshots [15], have been collected by evaluating the structural response for a number of parameter sets chosen according to, e.g., the latin hypercube sampling or sparse grid techniques. The RB functions are then generated by orthonormalization of the snapshots with respect to a suitable scalar product. 
Focusing finally on the parametrization of the elastodynamic problem, structural damage has been accounted for via non-homogeneity of the material stiffness, which features an explicit dependence on the placement. To build the dataset for the training of the classifier, the number of damage scenarios has been fixed as finite. This set has to cover data, like the most probable regions where a reduction in stiffness is expected to take place, to be chosen on the basis of a statistical analysis of the structural behavior.

\section{Numerical Results}

To start assessing the performance of the proposed approach, a cantilever beam of length $L=4 \mathrm{~m}$ and with an IPE 200 cross-section has been considered. The beam is made of steel, with a density $\varrho=7800 \mathrm{~kg} / \mathrm{m}^{3}$. A schematic representation of the problem is reported in Figure 1: vibrations of the cantilever are due to a surface load $g^{3}$, assumed constant over $x$ and with a sinusoidal variation in time.

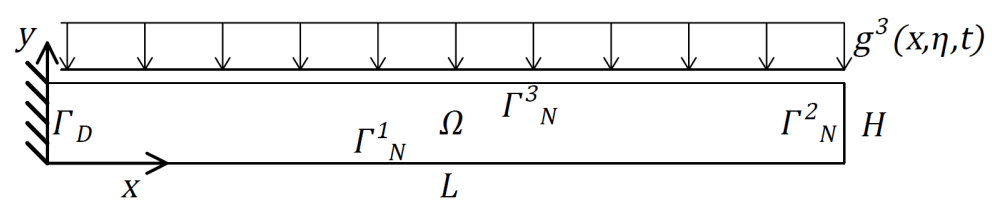

Figure 1. Schematic representation of the cantilever beam problem.

To mimic a space-varying damage pattern, the domain $\Omega$ has been subdivided into four subdomains $\Omega^{r}$, with $r=1, \ldots, 4$, of equal size and wherein the Young's modulus has been assumed to be constant. In the dataset construction, uncertainties have been taken into account through a parametrization of the loading condition (regarding both its amplitude and frequency) and of the damage distribution or variation of the current structural stiffness. Modeling and damage uncertainties must be therefore addressed by associating probabilistic distributions to characterize the variability of the geometric, material and loading properties. Specifically, uniform constrained distributions have been adopted for the amplitude and frequency of the distributed load. Regarding instead the damage uncertainty, five damage scenarios (baseline undamaged state, plus the four cases characterized by one single subdomain damaged) have been allowed for with the same probability: in all the damage scenarios, a stiffness reduction by $25 \%$ has been enforced in a single subdomain.

The FCN has been trained with 20,480 beam responses, each one lasting $1.2 \mathrm{~s}$ and recorded with a sampling rate of $667 \mathrm{~Hz}$. The deflection at the points on the top surface of the cantilever located at the interface between adjacent domains $\Omega^{r}$, has been specifically recorded. Each damage scenario has been simulated 4096 times through a ROM of the structure, which enabled to take into account 12 modes only in place of the 1002 degrees of freedom of the high-fidelity finite element (FE) model of the cantilever. Figure 2 shows the evolution of the training and validation loss for the training dataset. In these plots, the iteration number accounts for the epochs as well as for the batch size: if the dataset is composed by 100 instances and a batch size of 10 instances is adopted, after the first epoch the iteration number then amounts to 10. Once trained, the FCN has been tested on 320 cases generated via the FE model: Figure 3 collects the obtained results, shown via the confusion matrix summing up the classification output. Though accuracy is reported to be not homogeneous for all the test cases, a remarkable overall classification accuracy of $93.1 \%$ has been obtained. 


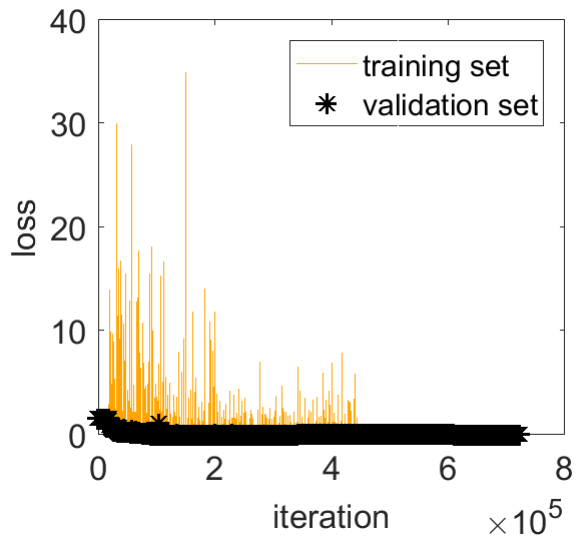

(a) Loss

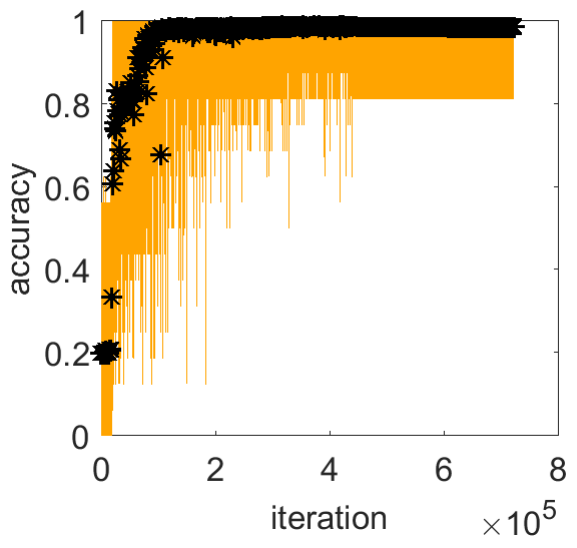

(b) Accuracy

Figure 2. Evolution of training and validation losses (a) and of training and validation accuracies (b).

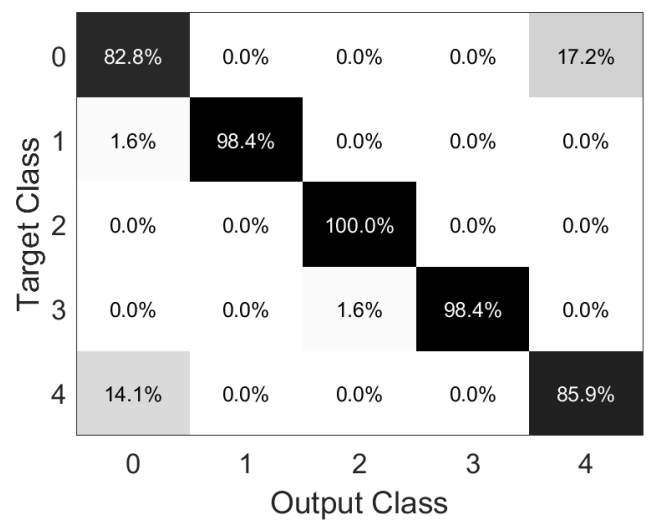

Figure 3. Confusion matrix relevant to damage localization.

\section{Conclusions}

In this work, we have discussed a hybrid model- and data-based approach to the health monitoring of civil structures. To enhance the performance of the procedure and move towards real-time monitoring, the high-fidelity physical models of the structures have been replaced by reduced-order ones, still guaranteeing a target accuracy of the solution. Damage detection has been framed as a classification problem: A set of solutions featuring different damage patterns or scenarios haven been considered, and environmental/loading variability has been also allowed for by stochastically varying the forcing terms. Deep learning has been finally exploited to train the classifier of the damage state.

Results relevant to a cantilever beam, featuring four different possible damage scenarios, have been used to assess the remarkable accuracy of the proposed method, basically stemming from the hybrid approach.

In future works, the capability of the SHM strategy will be further assessed in complex real-life cases. The architecture of the neural network/classifier will be also investigated, in order to check the effects of the relevant hyperparameters and of the stacking of convolutional layers on the performance of health monitoring.

Acknowledgments: The financial support from MIUR Project PRIN 15-2015LYYXA 8 “Multi-scale mechanical models for the design and optimization of microstructured smart materials and metamaterials" is gratefully acknowledged. 


\section{References}

1. Chang, P.C.; Flatau, A.; Liu, S.C. Health Monitoring of Civil Infrastructure. Struct. Health Monit. 2003, 2, 257-267.

2. Farrar, C.; Worden, K. Structural Health Monitoring A Machine Learning Perspective; Wiley: Hoboken, NJ, USA, 2013.

3. Capellari, G.; Eftekhar Azam, S.; Mariani, S. Damage detection in flexible plates through reduced-order modeling and hybrid particle-Kalman filtering. Sensors 2016, 16, 2.

4. Eftekhar Azam, S.; Mariani, S.; Attari, N. Online damage detection via a synergy of proper orthogonal decomposition and recursive Bayesian filters. Nonlinear Dyn. 2017, 89, 1489-1511.

5. Eftekhar Azam, S.; Mariani, S. Online damage detection in structural systems via dynamic inverse analysis: A recursive Bayesian approach. Eng. Struct. 2018, 159, $28-45$.

6. Farrar, C.R.; Doebling, S.W.; Nix, D.A. Vibration-Based Structural Damage Identification. Philos. Trans. Math. Phys. Eng. Sci. 2001, 359, 131-149.

7. Taddei, T.; Penn, J.; Yano, M.; Patera, A. Simulation-based classification; a model-order-reduction approach for structural health monitoring. Arch. Comput. Methods Eng. 2018, 25, $23-45$.

8. W. Doebling, S.; Farrar, C.; Prime, M. A Summary Review of Vibration-Based Damage Identification Methods. Shock Vib. Dig. 1998, 30, 91-105.

9. Eftekhar Azam, S.; Mariani, S. Dual estimation of partially observed nonlinear structural systems: A particle filter approach. Mech. Res. Commun. 2012, 46, 54-61.

10. Eftekhar Azam, S.; Mariani, S. Investigation of computational and accuracy issues in POD-based reduced order modeling of dynamic structural systems. Eng. Struct. 2013, 54, 150-167.

11. Sohn, H.; Worden, K.; Farrar, C.R. Statistical Damage Classification Under Changing Environmental and Operational Conditions. J. Intell. Mater. Syst. Struct. 2002, 13, 561-574.

12. Entezami, A.; Shariatmadar, H. Damage localization under ambient excitations and non-stationary vibration signals by a new hybrid algorithm for feature extraction and multivariate distance correlation methods. Struct. Health Monit. 2019, 18, 347-375.

13. Eftekhar Azam, S. Online Damage Detection in Structural Systems; Springer: Cham, Switzerland, 2014.

14. Milani, R.; Quarteroni, A.; Rozza, G. Reduced basis method for linear elasticity problems with many parameters. Comput. Methods Appl. Mech. Eng. 2008, 197, 4812-4829.

15. Quarteroni, A.; Manzoni, A.; Negri, F. Reduced Basis Methods for Partial Differential Equations: An Introduction; Springer: Cham, Switzerland, 2015; Volume 92.

16. Hinton, G.E.; Salakhutdinov, R.R. Reducing the Dimensionality of Data with Neural Networks. Science 2006, 313, 504-507.

17. Goodfellow, I.; Bengio, Y.; Courville, A. Deep Learning; MIT Press: Cambridge, MA, USA, 2016.

18. Pathirage, C.S.N.; Li, J.; Li, L.; Hao, H.; Liu, W.; Wang, R. Development and application of a deep learning-based sparse autoencoder framework for structural damage identification. Struct. Health Monit. 2019, 18, 103-122.

19. Choy, W.A. Structural Health Monitoring with Deep Learning. In Proceedings of the International MultiConference of Engineers and Computer Scientists, Hong Kong, China, 14-16 March 2018; pp. 557-560.

20. Capellari, G.; Chatzi, E.; Mariani, S. Structural Health Monitoring Sensor Network Optimization through Bayesian Experimental Design. ASME J. Risk Uncertain. Eng. Syst. Part A: Civ. Eng. 2018, 4, 04018016.

21. Capellari, G.; Chatzi, E.; Mariani, S. Cost-benefit optimization of structural health monitoring sensor networks. Sensors 2018, 18, 2174.

22. Rosafalco, L.; Manzoni, A.; Mariani, S.; Corigliano, A. Fully Convolutional Networks for Structural Health Monitoring through Multivariate Time Series Classification. arXiv 2020, arXiv:2002.07032.

23. Rosafalco, L.; Corigliano, A.; Manzoni, A.; Mariani, S. Combined Model Order Reduction and Artificial Neural Network for data assimilation and damage detection in structures. In Proceedings of the Computational Sciences and Artificial Intelligence in Industry (CSAI), Jyväskylä (Finland), 12-14 June 2019.

24. Karim, F.; Majumdar, S.; Darabi, H.; Harford, S. Multivariate LSTM-FCNs for time series classification. Neural Netw. 2019, 116, 237-245. 
25. Wang, Z.; Yan, W.; Oates, T. Time series classification from scratch with deep neural networks: A strong baseline. In Proceedings of the International Joint Conference on Neural Networks (IJCNN), Anchorage, AK, USA, 14-19 April 2017; pp. 1578-1585.

26. Abadi, M.; Agarwal, A.; Barham, P.; Brevdo, E.; Chen, Z.; Citro, C.; Corrado, G.S.; Davis, A.; Dean, J.; Devin, M.; et al. TensorFlow: Large-Scale Machine Learning on Heterogeneous Systems, 2015. Available online: tensorflow.org (accessed on 10 November 2019).

27. Haykin, S. Neural Networks and Learning Machines; Prentice Hall: Upper Saddle River, NJ, USA, 2009.

28. Ioffe, S.; Szegedy, C. Batch Normalization: Accelerating Deep Network Training by Reducing Internal Covariate Shift. arXiv 2015, arXiv:1502.03167.

29. Glorot, X.; Bengio, Y. Understanding the difficulty of training deep feedforward neural networks. In Proceedings of the Thirteenth International Conference on Artificial Intelligence and Statistics (AISTATS 2010), Sardinia, Italy, 13-15 May 2010; Volume 9, pp. 249-256.

2019 by the authors. Licensee MDPI, Basel, Switzerland. This article is an open access article distributed under the terms and conditions of the Creative Commons Attribution (CC BY) license (http://creativecommons.org/licenses/by/4.0/). 